\title{
Enhancement of tendon-bone healing following rotator cuff repair using hydroxyapatite with TGFß1
}

\author{
XIEBO YOU ${ }^{1}$, YUEQIN SHEN ${ }^{2}$, WEIHAN YU ${ }^{1}$ and YAOHUA HE ${ }^{1}$ \\ ${ }^{1}$ Department of Orthopedics, Shanghai Jiao Tong University Affiliated Sixth People's Hospital, Shanghai 200233; \\ ${ }^{2}$ State Key Laboratory of High Performance Ceramics and Superfine Microstructure, Shanghai Institute of Ceramics, \\ Chinese Academy of Science, Shanghai 200050, P.R. China
}

Received September 27, 2017; Accepted December 15, 2017

DOI: $10.3892 / \mathrm{mmr} .2018 .8499$

\begin{abstract}
The formation of fibrocartilage at the healing site following a rotator cuff tear repair is a major problem in the field of tendon-bone healing. The present study aimed to enhance the healing of the tendon-bone interface following rotator-cuff tear repair by the interposition of hydroxyapatite (HA) encapsulated with transforming growth factor $\beta 1$ (TGF $\beta 1$ ). Using an acute rotator cuff repair model, rats were divided into three groups: i) Repair only (control); ii) HA group; and iii) HA-TGF $\beta 1$ group. Animals were sacrificed at 2, 4 and 8 weeks following surgery. Micro-computed tomography (CT), histomorphometric analyses and biomechanical tests were used to evaluate the supraspinatus tendon-bone complex. The micro-CT images revealed notable novel bone formation in the groups treated with HA-TGF $\beta 1$. The histomorphometric analyses demonstrated improved fibrocartilage formation and collagen organization at the tendon-bone interface. The HA-TGF $\beta 1$ combination significantly improved the area of fibrocartilage, particularly at early time points ( 2 and 4 weeks). There was a significantly greater load-to-failure force achieved in the HA and HA-TGF $\beta 1$ groups compared with the control group at 4 and 8 weeks. Augmentation of the ceramic powder with HA-TGF $\beta 1$ at the tendon-bone interface was demonstrated to strengthen the healing entheses, increase bone and fibrocartilage formation and improve collagen organization compared with surgical repair alone. Local application of HA-TGF $\beta 1$ demonstrates potential in improving tendon-bone healing following rotator cuff repair.
\end{abstract}

Correspondence to: Dr Yaohua He, Department of Orthopedics, Shanghai Jiao Tong University Affiliated Sixth People's Hospital, 600 Yishan Road, Shanghai 200233, P.R. China

E-mail: heyaohua@sjtu.edu.cn

Key words: hydroxyapatite, transforming growth factor $\beta 1$, rotator cuff tear, tendon-bone healing

\section{Introduction}

A rotator cuff tear (RCT) is the one of the most common injuries that causes shoulder pain and dysfunction. Despite improvements in surgical techniques, firm attachment at the tendon-bone interface following rotator cuff repair remains a problem. Previous studies have reported high re-tear rates following rotator cuff repair (1-5). The re-tear of a rotator cuff negatively affects the long-term outcomes of patients compared with patients whose repairs heal without a recurrent defect (6). Therefore, improving tendon-bone healing following rotator cuff repair is a subject of interest.

In previous studies regarding tendon-bone healing enhancement, hydroxyapatite (HA) has been demonstrated to have positive effects in anterior cruciate ligament (ACL) reconstruction models $(7,8)$ as well as RCT repair models (9). Huangfu and Zhao (8) demonstrated that a mixture of tricalcium phosphate and HA led to better biomechanical results and more mature histological patterns compared with the control group. Li et al (7) demonstrated that an HA coating could induce polyethylene terephthalate artificial ligament graft osseointegration in the bone tunnel in a rabbit model. Zhao et al (9) applied $\mathrm{Ca}_{5}\left(\mathrm{PO}_{4}\right)_{2} \mathrm{SiO}_{4}(\mathrm{CPS})$ and $\mathrm{HA}$ at the interface of a rotator cuff repair site following a chronic RCT, and both CPS and HA bioceramics aided in cell attachment and proliferation, and accelerated novel bone formation. Ideally, HA should not only supply material for the reconstruction of the tendon-bone interface but also offer stimulatory information for cell proliferation and differentiation.

Tendons attach to the bone across fibrocartilaginous transition areas that can be divided into four distinct zones: Tendon, fibrocartilage, mineralized fibrocartilage and bone (10). The transforming growth factor $\beta$ (TGF $\beta$ ) family serves a key function in connective tissue development (11-13). Previous studies have investigated the roles of TGF $\beta 1$ and TGF $\beta 3$ in the process of healing following rotator cuff repair. Arimura et al (14) demonstrated that TGF $\beta 1$ enhances the formation of tough fibrous tissues at the healing site by inhibiting matrix metalloproteinase (MMP)-9 and MMP-13 expression to increase collagen accumulation but without the growth of tenogenic lineage cells. TGF $\beta 1$ has also been reported to be effective in enhancing the biomechanical properties of tendons or ligaments in rabbit and dog models $(15,16)$. By contrast, 
Manning et al (13) used an osmotic pump for sustained delivery of growth factors at the repair site and demonstrated that TGF $\beta 1$ promoted the formation of scar tissue. There was no improvement in healing with the application of TGF $\beta 3$. The role of TGF $\beta$ isoforms remains poorly characterized and requires further investigation.

The purpose of the present study was to evaluate whether the interposition of HA materials encapsulated with TGF $\beta 1$ could enhance the structural, histological and biomechanical properties in a rat rotator cuff repair model. The authors hypothesized that i) the interposition of HA during RCT repair would enhance the tendon-bone site healing; and ii) TGF $\beta 1$ would promote fibrocartilage formation, and improve the healing structure in the interface zone. Overall, the present study hypothesized that HA ceramics with TGF $\beta 1$ would have better characteristics compared with HA alone and demonstrate a better promoting effect in the interface area.

\section{Materials and methods}

Preparation of HA-TGF $\beta 1$. HA hierarchically nanostructured microspheres, which have potential applications in drug delivery and protein adsorption owing to their relatively large specific surface area, nanoporosity and hollow structure, were synthesized as reported by Qi et al (17). Briefly, $0.1110 \mathrm{~g} \mathrm{CaCl}_{2}$ and $0.1963 \mathrm{~g}$ creatine phosphate disodium salt tetrahydrate were dissolved in deionized water $(40 \mathrm{ml})$ with consistent magnetic stirring at room temperature while the $\mathrm{pH}$ value was maintained at 10 using $1 \mathrm{M}$ sodium hydroxide $(\mathrm{NaOH})$ solution. The resulting solution was transferred into a $60-\mathrm{ml}$ autoclave-safe container, sealed and microwave-heated in a microwave oven (MDS-6; Sineo Microwave Chemistry Technology, Co., Ltd., Shanghai, China) to $120^{\circ} \mathrm{C}$ and maintained at this temperature for $10 \mathrm{~min}$. Then, following cooling to room temperature, the product was collected by centrifugation at room temperature for $5 \mathrm{~min}(10,000 \mathrm{x} \mathrm{g})$, followed by washing several times with deionized water and ethanol and drying at $60^{\circ} \mathrm{C}$ for $24 \mathrm{~h}$.

To promote the recovery of the tendon-bone-interface, human TGF $\beta 1$ growth factor was loaded into prepared HA microspheres. A dried powder of HA (100 mg) was added into a PBS solution of TGF $\beta 1(1 \mu \mathrm{g} / \mathrm{ml}, 10 \mathrm{ml})$; afterward, the resulting suspension was continuously agitated at $37^{\circ} \mathrm{C}$ for $24 \mathrm{~h}$. Finally, the growth factor-loaded HA hierarchically nanostructured, porous microspheres $(10 \mu \mathrm{g} / 100 \mathrm{mg}, \mathrm{HA}-\mathrm{TGF} \beta 1)$ were obtained by freeze-drying.

Surgical technique. All experimental procedures were approved by the Institutional Animal Studies Committee of Shanghai Jiao Tong University Affiliated Sixth Hospital (Shanghai, China; no: DWLL2017-0304).

A total of 135 male Sprague-Dawley rats (obtained at 350-450 g, 2 months, Shanghai SIPPR-BK Laboratory Animal Co., Ltd., Shanghai, China) were used in this study. They were provided with fresh water and rat chow ad libitum and housed in a specific pathogen free environment (temperature: $25^{\circ} \mathrm{C}$; humidity: $50 \%$; light and dark cycle: $\left.12-\mathrm{h}\right)$. The surgical procedure was performed according to previously published study (9). The animals were randomly divided into one of three groups. In the control group, the tendon was repaired to its anatomic footprint using transosseous repair $(n=45)$. In the experimental groups, 90 rats underwent transosseous repair and were implanted with either HA ceramic powder $(n=45)$ or HA-TGF $\beta 1$ ceramic powder $(n=45)$ to augment the repair.

Following anesthesia, a longitudinal incision was made on the anterolateral aspect of the shoulder, splitting the deltoid muscle. The acromioclavicular joint was divided to allow visualization of the rotator cuff tendons. The anterior margin of the supraspinatus tendon was identified adjacent to the biceps tendon and the posterior margin was determined by the junction with the infraspinatus tendon fibers. The supraspinatus tendon was marked with a 5-0 polypropylene suture (Ethicon, Inc., Somerville, NJ, USA). The tendon was then sharply dissected from its insertion site at the great tuberosity. The tuberosity was gently decorticated and debrided of all soft tissue and fibrocartilage with a scalpel blade until bleeding was noted. A $0.8-\mathrm{mm}$ anterior-posterior transverse bone tunnel was created at the humeral head. A small trough was made using an 18-gauge blunt needle located centrally in the footprint to allow for seating of the ceramic powder. For the control group, a Mason-Allen stitch using a 3-0 Ethibond ${ }^{\circledR}$ (Ethicon, Inc.) suture was placed into the supraspinatus tendon. Suture ends from the tendon were then passed through the bone tunnels and firmly tied over the humeral metaphyseal cortex, anatomically repairing the supraspinatus tendon to its native footprint. For the experimental groups, $2 \mathrm{mg} \mathrm{HA}$ or HA-TGF $\beta 1$ powder was implanted into the trough, while the trough remained empty in the control group. The deltoid muscle and skin were then closed, and the rats were allowed to engage in unrestricted cage activity. For 3 days postoperatively, buprenorphine $(0.05 \mathrm{mg} / \mathrm{kg})$ was administered subcutaneously for postoperative analgesia.

Gross observation. Animals were sacrificed at 2,4 and 8 weeks following surgery. Following euthanasia and dissection, all specimens were evaluated for macroscopic tendon-bone repair, residual materials, synovial hyperplasia and shoulder range of motion.

Micro-computed tomography (CT) analysis. A total of 45 animals (5 animals per group per time point) were sacrificed for micro-CT analysis at 2, 4 and 8 weeks following surgery. Their right shoulders were dissected to include only the supraspinatus tendon-bone complex and the proximal third of the humerus for fixation in a 1:1 solution of ethanol and sterile water at room temperature for $24 \mathrm{~h}$. The bone density and novel bone formation were assessed with micro-CT (eXplore Locus SP; GE Healthcare, Chicago, IL, USA). Each sample was placed in the holder surrounded by ethanol solution and scanned using the conditions of $80 \mathrm{kV}, 450 \mathrm{~mA}$ and a $0.045-\mathrm{mm}$ effective pixel size. The images were subjected to a global threshold to distinguish the bone voxels for each specimen. Following the threshold scan, the $3 \mathrm{D}$ reconstruction images were obtained. A customized $4 \times 4 \mathrm{~mm}^{2}$ cylindrical region of interest (ROI) was centered at the surface of the repaired supraspinatus tendon-bone footprint area, which contained the distal portion of the supraspinatus tendon and the bony footprint. The size of the ROI was determined based on post-mortem observations that the supraspinatus footprint measured $\sim 3.5 \times 3.5 \mathrm{~mm}^{2}$. The 
bone mineral density (BMD) and bone volume fraction (bone volume/total volume; BV/TV) of the ROI were calculated.

Histomorphometric analysis. A total of 45 animals were sacrificed for histomorphometric analysis. Following the necropsies, the tissue specimens were fixed in $10 \%$ neutral buffered formalin at room temperature for $48 \mathrm{~h}$. The tissue specimens were decalcified with Immunocal (Wuhan Servicebio Co., Ltd., Wuhan, China) and embedded in paraffin. Sections $(5-\mu \mathrm{m})$ of the repaired supraspinatus tendon-greater tuberosity construct were cut in the coronal plane. The tissue sections were stained with hematoxylin \& eosin, safranin O/fast green, and picrosirius red. Hematoxylin \& eosin staining were performed at room temperature for $3 \mathrm{~h}$. Safranin O/fast green were performed at room temperature for $2 \mathrm{~h}$. Picrosirius red staining were performed at room temperature for $3 \mathrm{~h}$.

Using light microscopy (Leica DM4000B; Leica Microsystems GmbH, Wetzlar, Germany), tissue sections stained with safranin $\mathrm{O} /$ fast green were analyzed to determine the total area of novel fibrocartilage formation at the tendon-bone interface of the RCT. Digital images were acquired by a Leica DFC420C camera (Leica Microsystems $\mathrm{GmbH}$ ). The ImageJ software program version 1.51 (National Institutes of Health, Bethesda, MD, USA) was used to outline the area of metachromasia on the safranin $\mathrm{O}$-stained slides at a total magnification of $\mathrm{x} 40$ in order to determine the area of novel fibrocartilage formation. The total area of metachromasia for each specimen was recorded for analysis.

Using polarized light microscopy, tissue sections were stained with picrosirius red for semi-quantitative analysis of the collagen deposition and maturation at the repair site. Measurements were obtained by rotating the polarization plane until maximum brightness was obtained to control the variations in specimen orientation on the slide. All the tissue sections were cut to a uniform thickness and the light intensities were measured under the same conditions of illumination and with the same settings to facilitate comparisons between groups. The digital images were imported into ImageJ for processing. The images underwent 8-bit digitization, producing images in which non-collagenous material was dark (zero) and collagenous material was depicted by gray scales from 1 to 255 . Ten rectangular areas (50x50 $\mu \mathrm{m})$ were randomly selected at the tendon near the interface region and the gray scales were measured and recorded.

Biomechanical testing. A total of 45 animals were sacrificed for biomechanical testing. The humerus with the attached supraspinatus tendon was dissected from the surrounding tissues. The cross-sectional area of the supraspinatus tendon at its insertion site was measured using a digital caliper. The tendon was then placed into a custom-designed uniaxial testing system. The tendon was secured in a screw grip while the humerus was secured into a vice grip. The specimen was preloaded to $0.1 \mathrm{~N}$ and then loaded to failure at a rate of $14 \mu \mathrm{m} / \mathrm{sec}$, corresponding to $0.4 \%$ strain. The maximum load at failure and the failure site were recorded. The ultimate stress at failure was calculated by dividing the ultimate load-to-failure by the cross-sectional area.

Statistical analysis. Data are presented as the mean \pm standard deviation. Statistical analysis was performed with SPSS 17.0
(SPSS Inc., Chicago, IL, USA) Statistical analysis was performed using one-way analysis of variance with the least significant difference method as a post hoc test to determine the level of significance. $\mathrm{P}<0.05$ was considered to indicate a statistically significant difference.

\section{Results}

Macroscopic observation. In all rats, the repaired tendons remained in continuity with the bones and no evidence of infection was observed at the surgical site. There was no obvious limit to the shoulder range of motion. There were no obvious differences in the gross appearance of tendons following sacrifice. In the HA and HA-TGF $\beta 1$ groups, the shoulders contained remnants of the ceramic powder at the tendon attachment site in the 2- and 4-week group. There were no remnants in the control group or in the experimental groups at the 8-week time point.

Micro-CT analysis. The BMD values of the HA-TGFb1 groups were significantly higher compared with the control group at all time points [ 2 weeks: $387.20 \pm 8.68 \mathrm{mg} / \mathrm{mm}^{3}$ (control) vs. $401.93 \pm 11.70 \mathrm{mg} / \mathrm{mm}^{3}$ (HA), and $404.73 \pm 0.87 \mathrm{mg} / \mathrm{mm}^{3}$ (HA-TGF $\beta 1$ ), $\mathrm{P}=0.025 ; 4$ weeks: $396.07 \pm 2.63 \mathrm{mg} / \mathrm{mm}^{3}$ (control) vs. $408.67 \pm 2.71 \mathrm{mg} / \mathrm{mm}^{3}$ (HA), $\mathrm{P}=0.040$, and $411.87 \pm 9.1 \mathrm{mg} / \mathrm{mm}^{3}$ (HA-TGF $\beta 1, \mathrm{P}=0.045$ ); 8 weeks: $410.00 \pm 7.88 \mathrm{mg} / \mathrm{mm}^{3}$ (control) vs. $423.00 \pm 4.01 \mathrm{mg} / \mathrm{mm}^{3}$ (HA), and $432.03 \pm 9.17 \mathrm{mg} / \mathrm{mm}^{3}$ (HA-TGF $\beta 1$ ), $\mathrm{P}=0.034]$. However, there were no significant differences between the experimental groups within the same time point (Fig. 1).

The same trend was not observed for the value of BV/TV. No significant differences in BV/TV values were observed among the control and experimental groups (Fig. 1). Otherwise, some remnants of powder at the tendon-bone insertion site were observed at 2 and 4 weeks following surgical repair in the experimental groups only.

Histological analysis. In the 2-week groups, the specimens in the HA and HA-TGF $\beta 1$ groups demonstrated that the tendon-bone interface had more organized collagen fibers compared with those in the control group. However, a mass of inflammatory cells, primarily consisting of polymorphonuclear leukocytes, was present in the HA group. In addition, chondrocytes and novel fibrocartilage formation was observed in the HA-TGF $\beta 1$ group (Fig. 2).

In the 4-week groups, better-oriented collagen fibers, fewer inflammatory cells and more bone ingrowth into the interface area could be observed in all groups compared with those at 2 weeks. In addition, a larger area of fibrocartilage was observed in the HA and HA-TGF $\beta 1$ groups compared with the control group (Fig. 2).

The specimens in the 8-week control group demonstrated better-organized fibers in the gap tissue. The fibers were in the same direction of tensile pull of the tendon compared with those in the 4-week control group. In addition, the fibrocartilage in the tendon-bone interface of the experimental groups was remodeled into a more natural appearance and increased novel bone formation was observed (Fig. 2).

Metachromasia. At all time points (2,4 and 8 weeks) following surgery, HA-TGF $\beta 1$ powder and HA powder significantly 

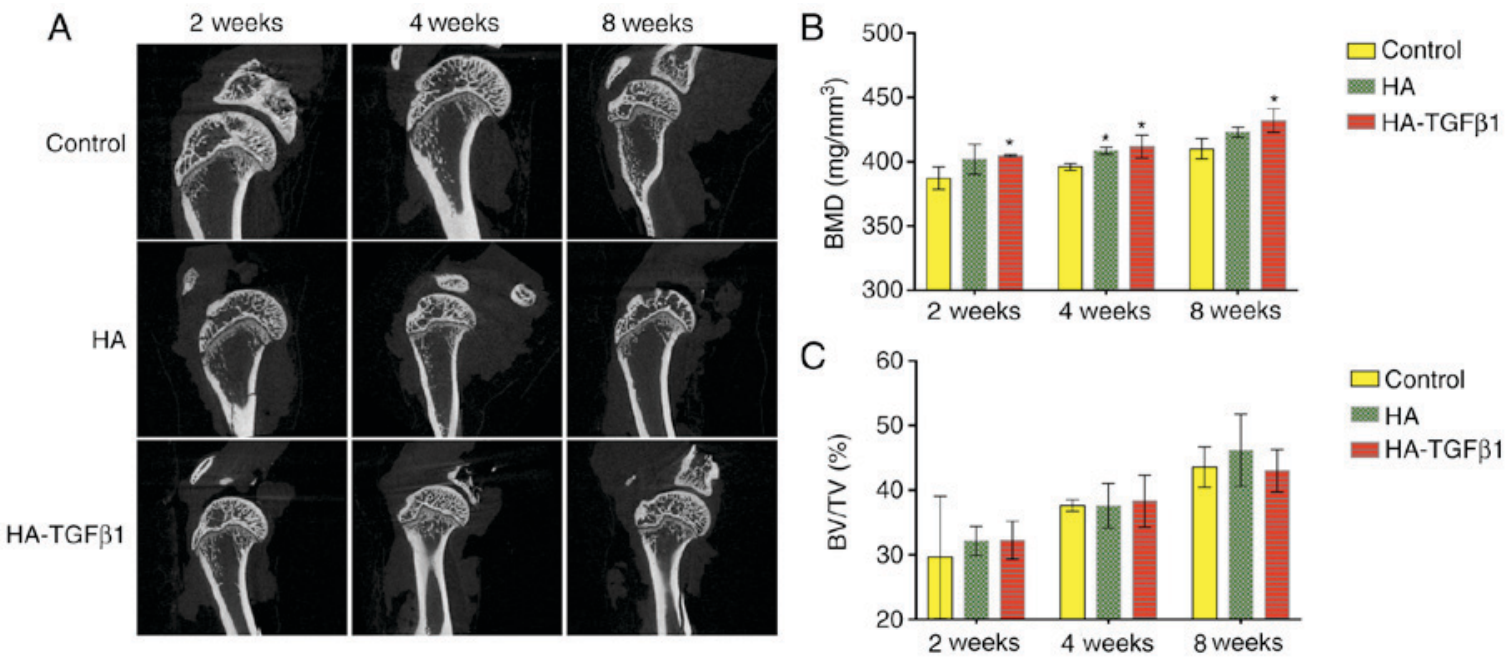

Figure 1. Analysis of the structure of the proximal humerus. (A) Micro-CT images of the proximal humerus. (B) Analysis of BMD. (C) Analysis of BV/TV. Data are presented as the mean \pm standard deviation ( $\mathrm{n}=5$ for each group). ${ }^{*} \mathrm{P}<0.05$ vs. control. HA-TGF $\beta 1$, hydroxyapatite-transforming growth factor $\beta 1$; $\mathrm{CT}$, computed tomography; BMD, bone mineral density; BV/TV, bone volume/total volume.

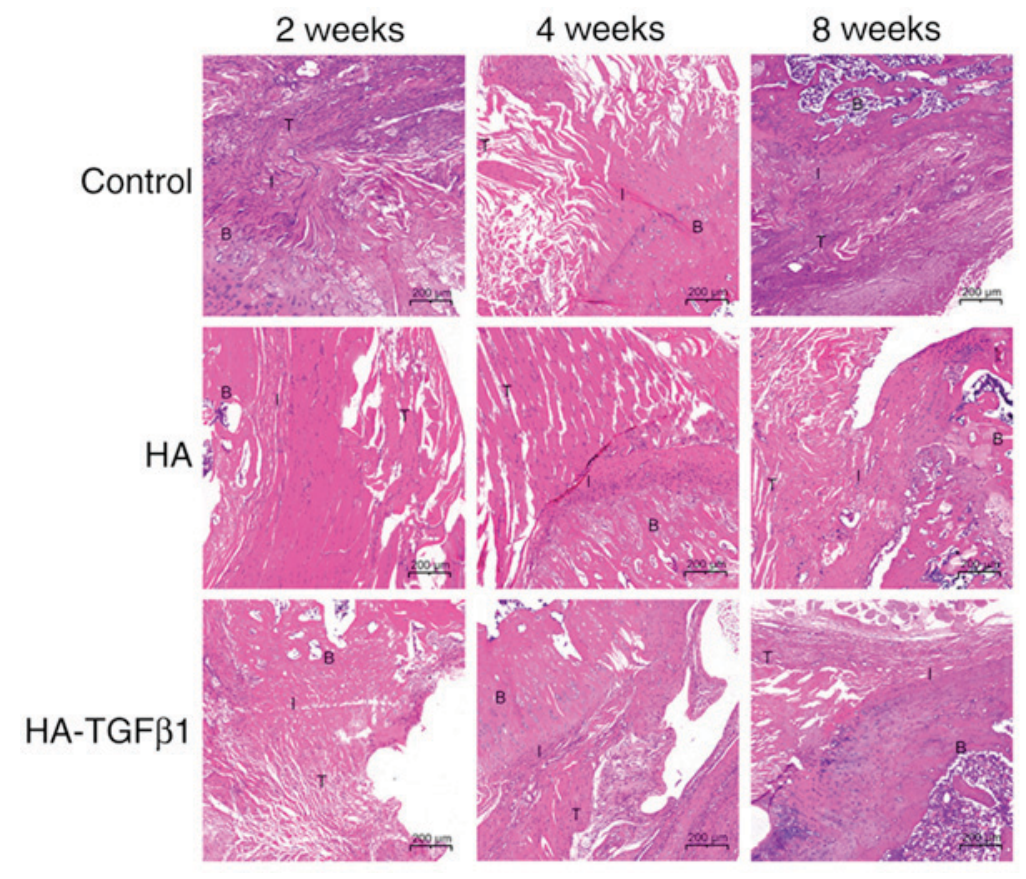

Figure 2. Hematoxylin and eosin-stained tissue sections of the healing enthesis at 2, 4 and 8 weeks postoperatively. B, bone; I, interface; T, tendon; HA, hydroxyapatite; HA-TGF $\beta 1$, hydroxyapatite-transforming growth factor $\beta 1$.

increased the area of glycosaminoglycan staining at the interface area compared with the control group [2 weeks: $113,928.2 \pm 14,736.7 \mu \mathrm{m}^{2}$ (control) vs. 152,623 $\pm 34,236 \mu \mathrm{m}^{2}$ (HA) $\mathrm{P}=0.049$ and $211,810 \pm 33,514 \mu \mathrm{m}^{2}$ (HA-TGF $\left.\beta 1\right) \mathrm{P}<0.05$; 4 weeks: $136,111 \pm 67,093 \mu \mathrm{m}^{2}$ (control) vs. $206,826 \pm 11,452 \mu \mathrm{m}^{2}$ (HA) $\mathrm{P}=0.049$ and $237,829 \pm 21,610 \mu \mathrm{m}^{2}$ (HA-TGF $\left.\beta 1\right) \mathrm{P}=0.012$; 8 weeks: $186,792 \pm 17,931 \mu \mathrm{m}^{2}$ (control) vs. $229,105 \pm 36,367 \mu \mathrm{m}^{2}$ (HA) $\mathrm{P}=0.031$ and 255,690 $\pm 24,626 \mu \mathrm{m}^{2}(\mathrm{HA}-\mathrm{TGF} \beta 1)$ $\mathrm{P}=0.002]$. The group implanted with HA-TGF $\beta 1$ powder demonstrated a significantly larger area of metachromasia $(\mathrm{P}<0.05)$ and a larger area of newly formed fibrocartilage in most specimens compared with the HA group at 2 and 4 weeks postoperatively. However, at 8 weeks, there were no significant differences between the HA and HA-TGF $\beta 1$ groups (Fig. 3).
Collagen organization. According to birefringence under polarized light at the tendon-bone-interface, the collagen organization in the experimental groups was improved significantly compared to that in the control group [2 weeks: $25.7 \pm 8.7$ grayscale units (control) vs. $37.4 \pm 13.4$ grayscale units (HA) $\mathrm{P}=0.027$ and $51.4 \pm 11.1$ grayscale units $(\mathrm{HA}-\mathrm{TGF} \beta 1) \mathrm{P}<0.05$; 4 weeks: $57.6 \pm 13.5$ grayscale units (control) vs. $73.3 \pm 17.9$ grayscale units $(\mathrm{HA}) \mathrm{P}=0.04$ and $97.9 \pm 32.3$ grayscale units (HA-TGF $\beta 1$ ) $\mathrm{P}=0.003$; 8 weeks: $104.3 \pm 29.0$ grayscale units (control) vs. $132.7 \pm 28.0$ grayscale units $(\mathrm{HA}), \mathrm{P}=0.039$ and $168.1 \pm 44.8$ grayscale units $(\mathrm{HA}-\mathrm{TGF} \beta 1), \mathrm{P}=0.001]$. In addition, collagen organization was significantly improved in the HA-TGF $\beta 1$ group compared with the HA group at 2, 4, and 8 weeks ( 2 weeks: $\mathrm{P}=0.021$; 4 weeks: $\mathrm{P}=0.049 ; 8$ weeks: 
A

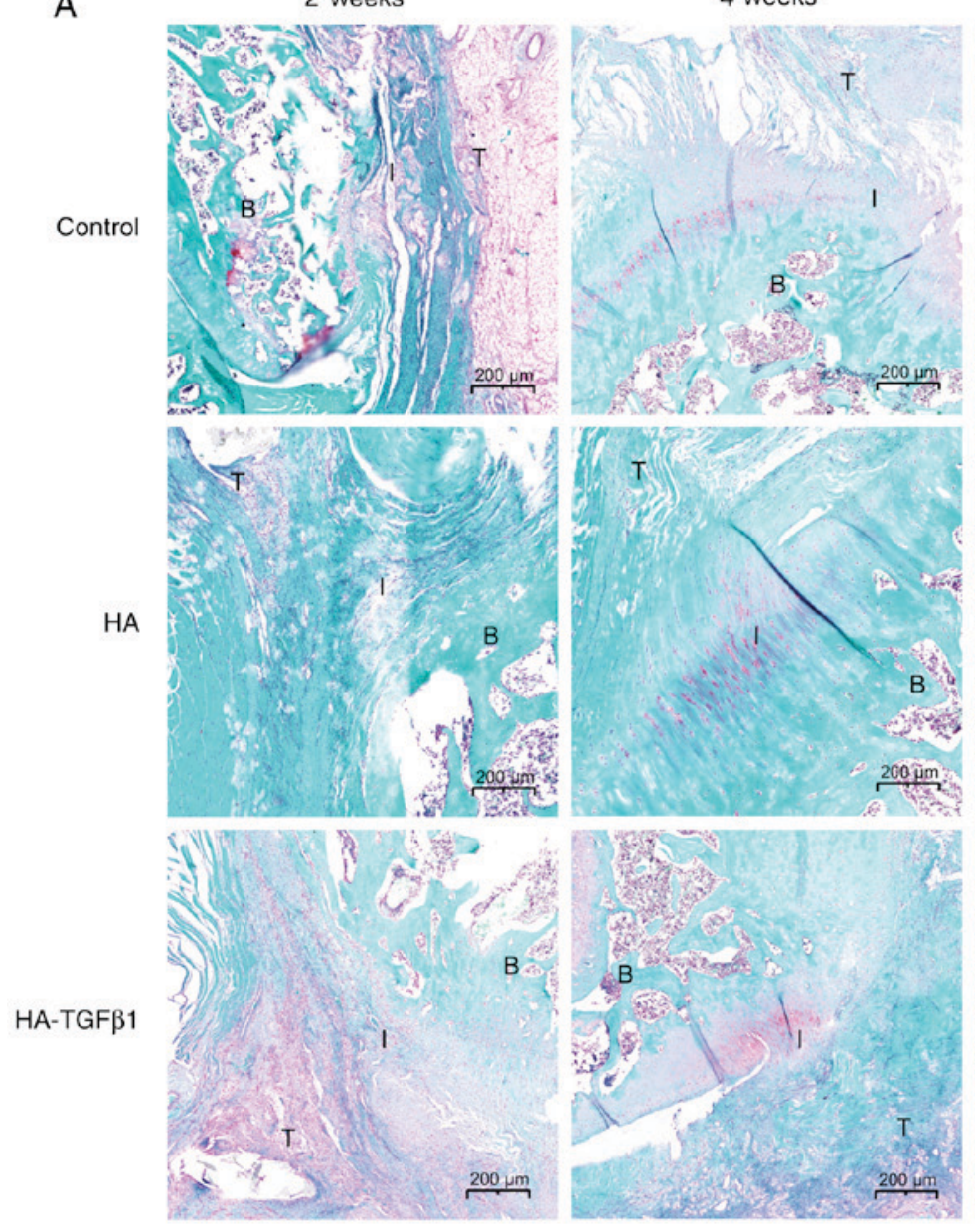

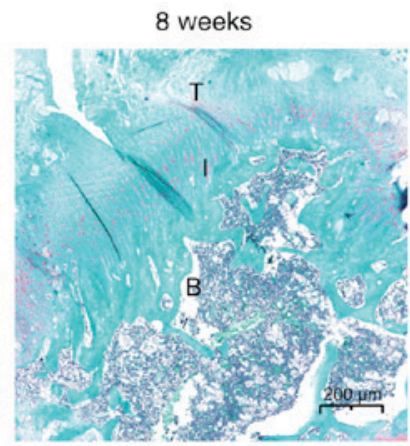
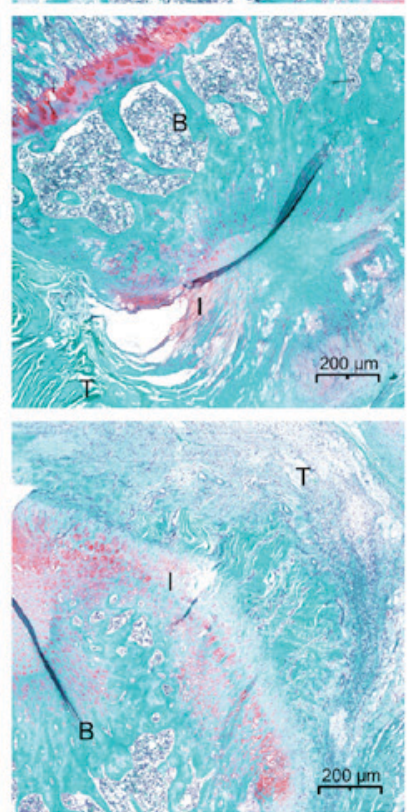

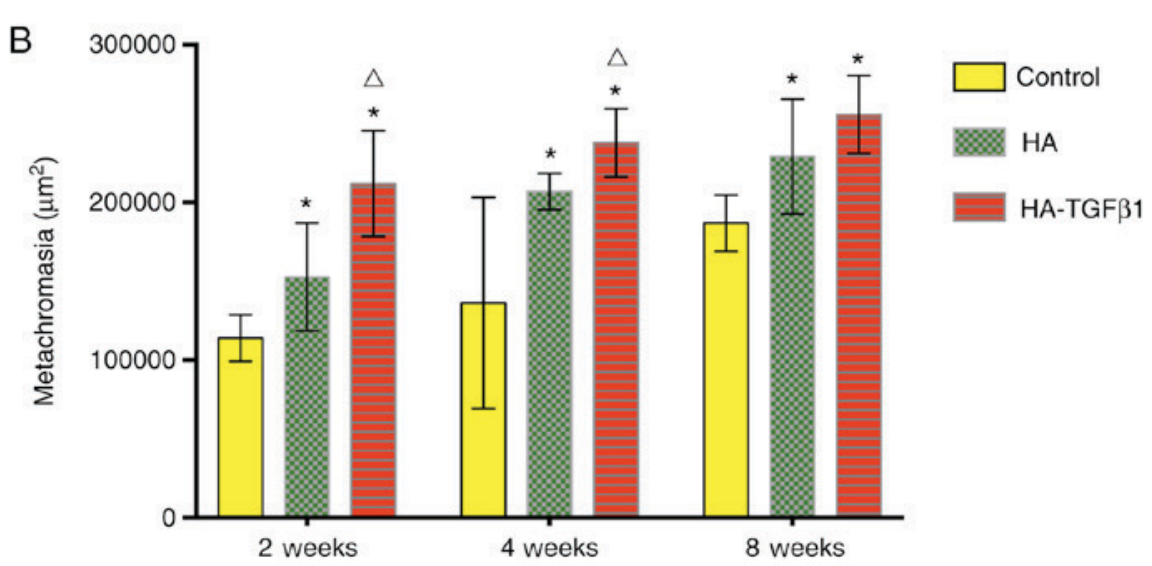

Figure 3. Histological analysis of the healing enthesis. (A) Representative safranin O-stained tissue sections of the healing enthesis at 2 , 4 and 8 weeks postoperatively. (B) Area of cartilage at the interface area with safranin O-stained slides. Data are presented as the mean \pm standard deviation ( $\mathrm{n}=5$ for each group). ${ }^{*} \mathrm{P}<0.05$ vs. control; ${ }^{\Delta} \mathrm{P}<0.05$ vs. HA. B, bone; I, interface; $\mathrm{T}$, tendon; HA-TGF $\beta 1$, hydroxyapatite-transforming growth factor $\beta 1$.

$\mathrm{P}=0.048)$. These results demonstrated the beneficial effect of $\mathrm{HA}$ and TGF $\beta 1$ on collagen production at the tendon-bone interface (Fig. 4).

Ultimate load-to-failure. There were no significant differences in the cross-sectional area of the healing enthesis between the control group and the experimental groups at 2, 4, or 8 weeks following surgery (Fig. 5).

At 2 weeks following surgery, no significant differences in the ultimate load-to-failure were observed (control: $8.2 \pm 0.4 \mathrm{~N}$; HA: $8.4 \pm 0.4 \mathrm{~N}$; HA-TGF $\beta 1: 8.4 \pm 0.3 \mathrm{~N}$ ). At 4 weeks, the ultimate load-to-failure forces in the experimental groups were significantly increased compared with the control group (control: $15.9 \pm 1.4 \mathrm{~N}$; HA: 18.1 $\pm 1.0 \mathrm{~N}, \mathrm{P}=0.02$; HA-TGF $\beta 1$ : $19.4 \pm 0.6 \mathrm{~N}, \mathrm{P}=0.001)$. At 8 weeks following surgery, the ultimate load-to-failure forces were significantly increased in the HA group and the HA-TGF $\beta 1$ group compared with the control group (control: $26.1 \pm 0.8 \mathrm{~N}$; HA: $29 \pm 1.3 \mathrm{~N}, \mathrm{P}=0.002$; HA-TGF $\beta 1: 30.4 \pm 1.1 \mathrm{~N}, \mathrm{P}<0.05)$. No significant differences in ultimate load-to-failure force were observed between the HA and HA-TGF $\beta 1$ groups at all time points postoperatively (Fig. 5). 
A
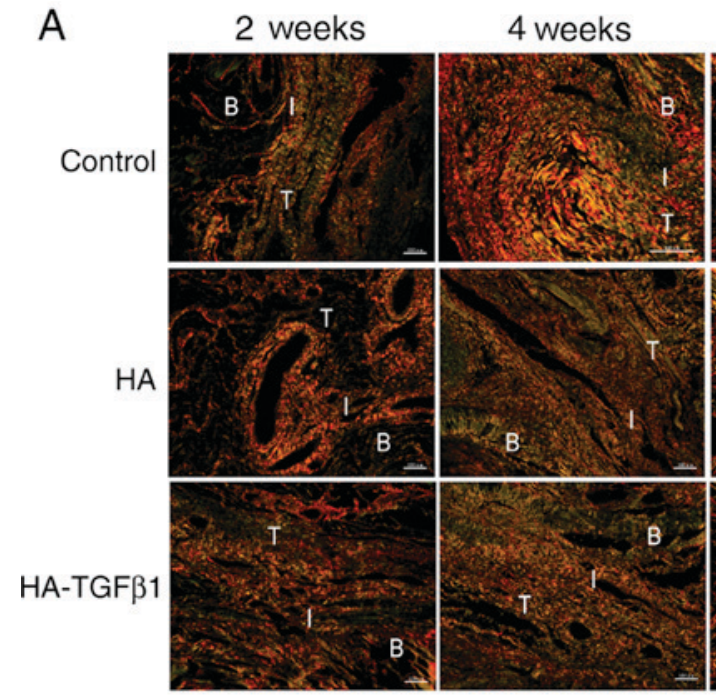

8 weeks

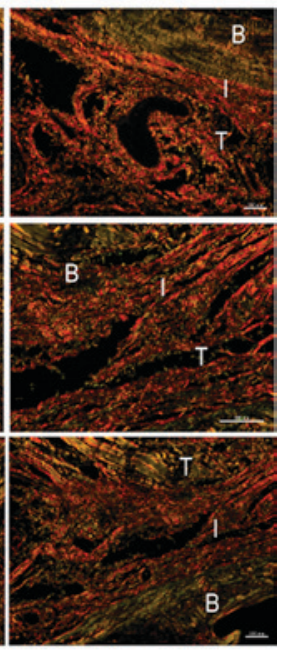

B

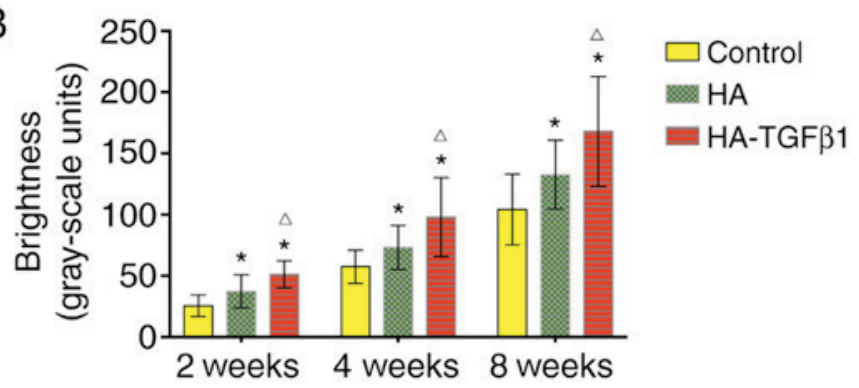

Figure 4. Further histological analysis of the healing enthesis. (A) Representative spicrosirius red-stained tissue sections of the healing enthesis at 2, 4 and 8 weeks postoperatively. (B) Area of collagen birefringence. Scale bar $=100 \mu \mathrm{m}$. Data are presented as the mean \pm standard deviation ( $\mathrm{n}=5$ for each group). "P $<0.05$ vs. control; ${ }^{\wedge} \mathrm{P}<0.05$ vs. HA. B, bone; I, interface; T, tendon; HA-TGF $\beta 1$, hydroxyapatite-transforming growth factor $\beta 1$.
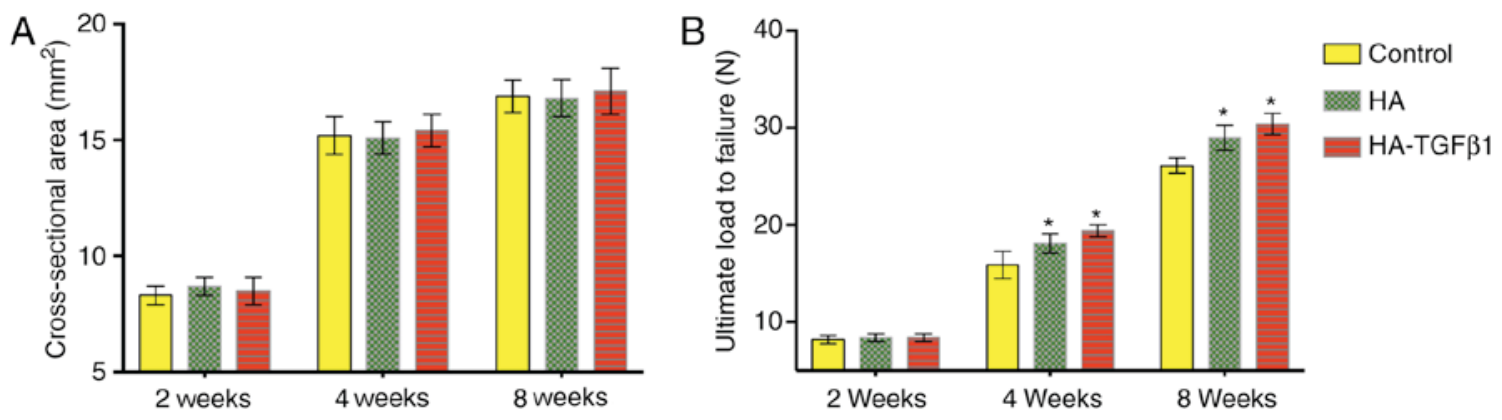

Figure 5. Biomechanical test of the tendon-bone interface. (A) Cross-sectional areas. (B) Ultimate load to failure. Data are presented as the mean \pm standard deviation ( $\mathrm{n}=5$ for each group). ${ }^{*} \mathrm{P}<0.05$ vs. control. HA-TGF $\beta 1$, hydroxyapatite-transforming growth factor $\beta 1$.

\section{Discussion}

The current study investigated whether the interposition of HA encapsulated with TGF $\beta 1$ could enhance the healing of the tendon-bone interface and promote bone and collagen formation following RCT repair in an acute rotator cuff injury model. The results demonstrate that the application of bioceramics may promote novel bone formation at the repair site. Micro-CT analysis revealed that HA bioceramics alone and with TGF $\beta 1$ exhibited obvious osteogenic activity and osteoconductivity at 2, 4 and 8 weeks post-surgery. The local application of the growth factor encapsulated into the HA was associated with the improvement of fibrocartilage and collagen formation at the tendon-bone interface compared with rotator cuff repair alone at all time points. This demonstrated a notable effect of this material in tendon-bone healing. Furthermore, the application of HA bioceramics alone improved the area of fibrocartilage and collagen organization compared with the control group. These results indicate that HA is a suitable drug carrier material in tendon-bone healing. At the 2 weeks after surgery there were improvements in the area of fibrocartilage and in collagen formation with HA-TGF $\beta 1$ bioceramics compared with HA bioceramics or repair only, which indicated that local delivery of this growth factor could enhance the mature healing enthesis at the tendon-bone repair site.

HA has been used as an osteoconductive material for bone growth. HA-based biomaterials are the most common materials used in modern bone substitution (18-21). In addition, 
a previous study revealed that tendon-bone healing depends upon the bone ingrowth into the healing interface (18). Zhao et al (9) demonstrated that the interposition of HA aids in cell attachment and proliferation, and accelerates novel bone formation. HA was selected for its osteoconductive character as the delivery vehicle for TGF $\beta 1$. HA was demonstrated to be an ideal augmentative matrix for rotator cuff repair with regards to cytocompatibility, osteogenic activity and osteoconductivity, according to a previous study (9). In the present study, HA was observed to be a suitable osteoconductive material, as it promoted increased bone formation at the footprint. HA could also enhance healing by increasing the area of fibrocartilage and improving collagen organization at the tendon-bone insertion. HA alone could serve a non-negligible role in the tendon-bone healing process.

A previous study demonstrated the potential of TGF $\beta 1$ in the application of tendon-bone healing. TGF $\beta 1$ could significantly increase the binding strength of the graft to the tunnel wall following ACL reconstruction (16). In addition, the perpendicular collagen fibers connecting the tendon to the bone were richly regenerated in the TGF $\beta 1$ group. Kovacevic et al (22) combined TGF 33 with an injectable $\mathrm{Ca}-\mathrm{P}$ matrix and demonstrated that it may improve healing following rotator cuff repair. A recent study demonstrated that upregulating TGF $\beta$ expression in bone mesenchymal stem cells (BMSCs) can promote tendon-to-bone healing following ACL reconstruction by regulating the TGF $\beta 1$ mitogen-activated protein kinase (MAPK) 1 signaling pathway (23). In addition, TGF $\beta 1$ is the most important fibrosis inducer (24), which could explain the increase in collagen fibers. Furthermore, TGF $\beta 1$ can also induce BMSC migration to bone resorption locations and promote bone resorption as well as formation (25). The micro-CT results of the present study also support this theory. The bone formation at the repair site increased in the HA-TGF $\beta 1$-treated repair group compared with repair only. In vitro research has indicated that MAPK subtypes regulate chondrogenesis of rat BMSCs by interaction with the TGF $\beta 1 /$ mothers against decapentaplegic homolog 3 signaling pathway (26). Using the rat rotator cuff repair model, the present study demonstrated that TGF $\beta 1$ delivery with an HA matrix could increase bone and fibrocartilage formation and improve collagen organization at the tendon-bone interface following rotator cuff repair.

There were several limitations of the present study. First, the acute injury and repair rat model is different from RCT and repair in humans. However, previous studies have validated the relevance of this animal model $(27,28)$. Second, the present study only investigated one dose of TGF $\beta 1$; this dose was selected according to a previous study in a rat rotator cuff repair model, where each animal received $2.75 \mu \mathrm{g}$ of TGF $\beta$ mixed with Ca-P matrix (22). Third, this is a pilot study using a small (size) animal model with limited evaluation tools and a limited observation period. Some differences observed may be relatively small. However, most differences were significant. In a future study, the authors plan to choose a large animal model that can simulate the human condition more accurately and to investigate the effect of multiple doses of TGF $\beta 1$, as well as multiple growth factors, on the healing process.

Local delivery of TGF $\beta 1$ in HA ceramic powder at the tendon-bone interface during rotator cuff repair was demonstrated to strengthen the healing enthesis, increase bone and fibrocartilage formation, and improve collagen organization compared with repair alone. In addition, the HA matrix itself was demonstrated to enhance the tendon-bone healing process. Further research should focus on loading HA ceramics with multiple growth factors. Augmentation of the repair combined with drug delivery may improve the clinical outcome of rotator cuff repair by enhancing the tendon-bone healing.

\section{Acknowledgements}

Financial support from the National Natural Science Foundation of China (grant nos. 81271961 and 81572106) is gratefully acknowledged.

\section{References}

1. Domb BG, Glousman RE, Brooks A, Hansen M, Lee TQ and ElAttrache NS: High-tension double-row footprint repair compared with reduced-tension single-row repair for massive rotator cuff tears. J Bone Joint Surg Am 90 (Suppl 4): S35-S39, 2008.

2. Nelson CO, Sileo MJ, Grossman MG and Serra-Hsu F: Single-row modified mason-allen versus double-row arthroscopic rotator cuff repair: A biomechanical and surface area comparison. Arthroscopy 24: 941-948, 2008.

3. Ozbaydar M, Elhassan B, Esenyel C, Atalar A, Bozdag E, Sunbuloglu E, Kopuz N and Demirhan M: A comparison of single-versus double-row suture anchor techniques in a simulated repair of the rotator cuff: An experimental study in rabbits. J Bone Joint Surg Br 90: 1386-1391, 2008.

4. Boileau P, Brassart N, Watkinson DJ, Carles M, Hatzidakis AM and Krishnan SG: Arthroscopic repair of full-thickness tears of the supraspinatus: does the tendon really heal? J Bone Joint Surg Am 87: 1229-1240, 2005.

5. Gerber C, Fuchs B and Hodler J: The results of repair of massive tears of the rotator cuff. J Bone Joint Surg Am 82: 505-515, 2000.

6. Zumstein MA, Jost B, Hempel J, Hodler J and Gerber C: The clinical and structural long-term results of open repair of massive tears of the rotator cuff. J Bone Joint Surg Am 90: 2423-2431, 2008.

7. Li H, Ge Y, Wu Y, Jiang J, Gao K, Zhang P, Wu L and Chen S: Hydroxyapatite coating enhances polyethylene terephthalate artificial ligament graft osseointegration in the bone tunnel. Int Orthop 35: 1561-1567, 2011.

8. Huangfu $\mathrm{X}$ and Zhao J: Tendon-bone healing enhancement using injectable tricalcium phosphate in a dog anterior cruciate ligament reconstruction model. Arthroscopy 23:455-462, 2007.

9. Zhao S, Peng L, Xie G, Li D, Zhao J and Ning C: Effect of the interposition of calcium phosphate materials on tendon-bone healing during repair of chronic rotator cuff tear. Am J Sports Med 42: 1920-1929, 2014.

10. Gulotta LV and Rodeo SA: Growth factors for rotator cuff repair. Clin Sports Med 28: 13-23, 2009.

11. Kim HM, Galatz LM, Das R, Havlioglu N, Rothermich SY and Thomopoulos $\mathrm{S}$ : The role of transforming growth factor beta isoforms in tendon-to-bone healing. Connect Tissue Res 52: 87-98, 2011.

12. Pryce BA, Watson SS, Murchison ND, Staverosky JA, Dünker N and Schweitzer R: Recruitment and maintenance of tendon progenitors by TGFbeta signaling are essential for tendon formation. Development 136: 1351-1361, 2009.

13. Manning CN, Kim HM, Sakiyamaelbert SE, Sakiyama-Elbert S, Galatz LM, Havlioglu N and Thomopoulos S: Sustained delivery of transforming growth factor beta three enhances tendon-to-bone healing in a rat model. J Orthop Res 29: 1099-1105, 2011.

14. Arimura H, Shukunami C, Tokunaga T, Karasugi T, Okamoto N, Taniwaki T, Sakamoto H, Mizuta H and Hiraki Y: TGF- $\beta 1$ improves biomechanical strength by extracellular matrix accumulation without increasing the number of tenogenic lineage cells in a rat rotator cuff repair model. Am J Sports Med 45: 2394-2404, 2017. 
15. Anaguchi Y, Yasuda K, Majima T, Tohyama H, Minami A and Hayashi K: The effect of transforming growth factor-beta on mechanical properties of the fibrous tissue regenerated in the patellar tendon after resecting the central portion. Clin Biomech 20: 959-965, 2005.

16. Yamazaki S, Yasuda K, Tomita F, Tohyama H and Minami A: The effect of transforming growth factor-betal on intraosseous healing of flexor tendon autograft replacement of anterior cruciate ligament in dogs. Arthroscopy 21: 1034-1041, 2005.

17. Qi C, Zhu YJ, Lu BQ, Zhao XY, Zhao J, Chen F and Wu J: Hydroxyapatite hierarchically nanostructured porous hollow microspheres: Rapid, sustainable microwave-hydrothermal synthesis by using creatine phosphate as an organic phosphorus source and application in drug delivery and protein adsorption. Chemistry 19: 5332-5341, 2013.

18. Galatz LM, Sandell LJ, Rothermich SY, Das R, Mastny A, Havlioglu N, Silva MJ and Thomopoulos S: Characteristics of the rat supraspinatus tendon during tendon-to-bone healing after acute injury. J Orthop Res 24: 541-550, 2006.

19. Canullo L, Heinemann F, Gedrange T, Biffar R and Kunert-Keil C: Histological evaluation at different times after augmentation of extraction sites grafted with a magnesium-enriched hydroxyapatite: Double-blinded randomized controlled trial. Clin Oral Implants Res 24: 398-406, 2013.

20. Hamerschmidt R, Santos RF, Araújo JC, Stahlke Jr HJ, Agulham MA, Moreira AT and Mocellin M: Hydroxyapatite granules used in the obliteration of mastoid cavities in rats. Braz J Otorhinolaryngol 77: 315-321, 2011 (In English, Portuguese).

21. Gredes T, Gedrange T, Hinuber C, Gelinsky $M$ and Kunert-Keil C: Histological and molecular-biological analyses of poly(3-hydroxybutyrate) (PHB) patches for enhancement of bone regeneration. Ann Anat 199: 36-42, 2015.
22. Kovacevic D, Fox AJ, Bedi A, Ying L, Deng XH, Warren RF and Rodeo SA: Calcium-phosphate matrix with or without TGF- $\beta 3$ improves tendon-bone healing after rotator cuff repair. Am J Sports Med 39: 811-819, 2011.

23. Wang R, Xu B and Xu HG: Up-regulation of TGF- $\beta$ promotes tendon-to-bone healing after anterior cruciate ligament reconstruction using bone marrow-derived mesenchymal stem cells through the TGF- $\beta /$ MAPK signaling pathway in a new zealand white rabbit model. Cell Physiol Biochem 41: 213-226, 2017.

24. Li B, Shao Q, Ji D, Li F and Chen G: Mesenchymal stem cells mitigate cirrhosis through BMP7. Cell Physiol Biochem 35: 433-440, 2015

25. Tang Y, Wu X, Lei W, Pang L, Wan C, Shi Z, Zhao L, Nagy TR, Peng $\mathrm{X}, \mathrm{Hu} \mathrm{J}$, et al: TGF-beta1-induced migration of bone mesenchymal stem cells couples bone resorption with formation. Nat Med 15: 757-765, 2009.

26. Li J, Zhao Z, Liu J, Huang N, Long D, Wang J, Li X and Liu Y: MEK/ERK and p38 MAPK regulate chondrogenesis of rat bone marrow mesenchymal stem cells through delicate interaction with TGF-beta1/Smads pathway. Cell Prolif 43: 333-343, 2010.

27. Carpenter JE, Thomopoulos S, Flanagan CL, DeBano CM and Soslowsky LJ: Rotator cuff defect healing: A biomechanical and histologic analysis in an animal model. J Shoulder Elbow Surg 7: 599-605, 1998.

28. Soslowsky LJ, Carpenter JE, Debano CM, Banerji I and Moalli MR: Development and use of an animal model for investigations on rotator cuff disease. J Shoulder Elbow Surg 5: 383-392, 1995.

This work is licensed under a Creative Commons Attribution-NonCommercial-NoDerivatives 4.0 International (CC BY-NC-ND 4.0) License. 